\title{
Reliability Evaluation of Parallel Redundancy Protocol Based Substation Process Bus
}

\author{
Jun $\mathrm{Mo}^{*}$ and Jiancheng Tan \\ Guangxi Key Laboratory of Power System Optimization and Energy Technology, Guangxi University, Nanning City 530004, \\ Guangxi Zhuang Autonomous Region, China \\ ${ }^{*}$ Corresponding author
}

\begin{abstract}
IEC 62439 defines the parallel redundancy protocol (PRP) which offers zero disruption time for network communication. This paper evaluates the reliability of PRP based substation process bus considering protection functions. Two typical process bus solutions are discussed for a typical transmission substation. To reduce the calculation complicity, the approximation algorithm of reliability evaluation is presented based on the direct disjoint algorithm of the minimal cut sets. An example for the bus protection is given to demonstrate the approximation algorithm. The comparison results show that the presented analysis method is simple and efficient for engineering system. Finally, the reliability and availability of the protection system architectures based on the two process bus solutions have been compared and some suggestions have been given for the application of process bus.
\end{abstract}

Keywords-parallel redundancy protocol (PRP); reliability; process bus; protection; sampled value (SV); generic objectoriented substation event (GOOSE)

\section{INTRODUCTION}

The network redundancy is widely used to improve the reliability of the IEC 61850 [1] based substation automation system (SAS). The process bus specified in IEC 61850-9-2 plays an important role in SAS communication. The bay level devices such as the protection and control devices or IEDs (Intelligent Electronic Devices) are connected to the process level devices such as merging units (MUs) and the intelligent switches over the process bus [2]. According to IEC 61850-5, process bus network redundancy needs to be approached not on a basis of failover times at all but on the basis of a seamless redundancy concept with no failover time at all. Since the conventional redundancy RSTP (Rapid Spanning Tree Protocol) cannot guarantee a zero or near-zero frame loss upon network failure occurrence, the Parallel Redundancy Protocol (PRP) and High availability Seamless Ring (HSR) speicified in IEC 62439 [3] have been recommend in the second edition of IEC 61850-9-2 part. Currently, one of most important issue left to substation design engineer is how to quantitatively evaluate the reliability of process bus based on PRP and/or HSR.

Some references [4-9] have provided the method to calculate reliability and availability of IEC 61850 based substation communication architectures. But most of them focus on the evaluation of network architectures rather than protection functions. The basic assumption that the failure modes are independent from each other may not be valid for the redundant system due to the common failure events which have been omitted by many papers. On the other hand, the conventional numerical analysis methods of network reliability, such as reliability block diagram (RBD), Markov, minimum path set method [10-13], still need improvement for complex problems [14]. For example, the Markov method is simple only for very simple models, while the complexity of solving Markov model grows exponentially as the sizes of the models grow linearly. RBD method is suitable for the serial-parallel system, but it is still difficult to draw the RBD for complex network topology architectures.

To address the above issues, this paper presents the direct disjoint form based reliability algorithm and performs the reliability evaluation of PRP based process bus considering protection functions. The comparison results show that the presented analysis method is simple and efficient for engineering system.

\section{PARALlEL REDUNDANCY PROTOCOL}

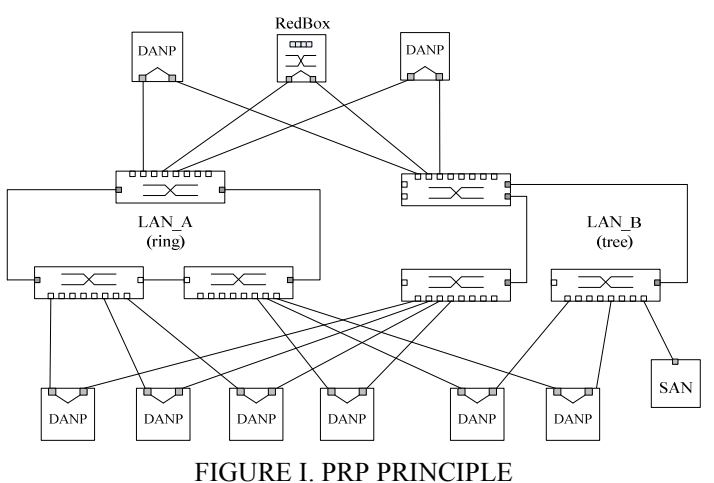

IEC 62439 defines the parallel pedundancy protocol which is a layer 2 redundancy protocol that provides seamless operation in case of loss of any link or switch. PRP relies on complete duplication of the LAN (see Figure 1). Both LANs operate in parallel and each individually can use RSTP. A source node (DANP) sends the same frame on both LANs and the destination nodes receive the first frame and discard the duplicate on the base of its source address and of a sequence number appended to the frame's payload. Singly Attached Node (SANs) that need to communicate with one another shall be attached to the same LAN or to both LANs through a RedBox. 


\section{Substation Process Bus}

\section{A. Study Case}

This paper considers the IEC 61850 classified T1-1 transmission substation as the reliability analytic case. As shown in Figure 2, it is $220 / 132 \mathrm{kV}$ small substation with single bus. There are total 5 bays, 3 line bays, one transformer bay and one bus bay. Each bay has redundancy protection IEDs (mian-1 and main-2).

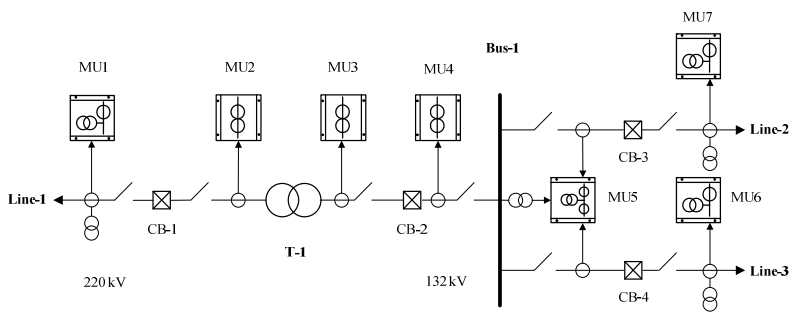

FIGURE II. T1-1 TRANSMISSION SUBSTATION

TABLE I. ARRANGEMENT OF IEDS AND MUS FOR PROTECTION FUNCTIONS

\begin{tabular}{ccc||ccc}
\hline Bay & $\begin{array}{c}\text { Prot. } \\
\text { IED }\end{array}$ & MU & Bay & Prot. IED & MU \\
\hline T-1 & 3,4 & 2,3 & Line-1 & 1,2 & 1 \\
$\begin{array}{c}\text { Bus- } \\
1\end{array}$ & 5,6 & 4,5 & Line-2 & 7,8 & 6 \\
- & - & - & Line-2 & 9,10 & 7 \\
\hline
\end{tabular}

For simplicity, all the IEDs and MUs have been numbered. The detailed configuration for each bay and the abbreviations for the components are tabulated in Table 1 and 2 respectively. It is very important to note that, the arrangement of IEDs and MUs depends on the practical requirement of substation, and this paper just performs the reliability evaluation for IEC 61850 process bus.

\section{B. Process Bus Solution}

IEC 61850 defines two important transmission models: sampled value (SV) and generic object-oriented substation event (GOOSE). SV model is used to implement the transmission of sampled values such as voltage and current signals. GOOSE model is responsible for the transmission of trip signals, control commends, status of disconnect switches and circuit breakers et al. There are two typical process bus solutions based on the application of SV and GOOSE

1) SV and GOOSE LAN Merge (PB solution \#1):

This process bus solution combines the takes full advantage of the network communication that SV and GOOSE messages are transmitted in the common LAN which could save the network cost for substation. For this solution, two Ethernet switches are required to achieve the PRP communication. Figure 3 shows the process bus architectures for Line-1, T-1 and Bus-1 bay.

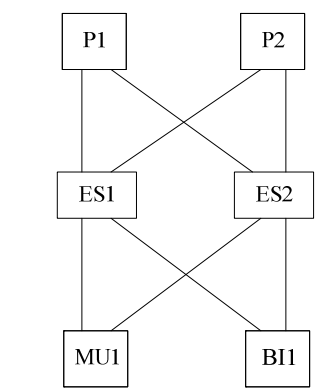

(a) Line-1 bay

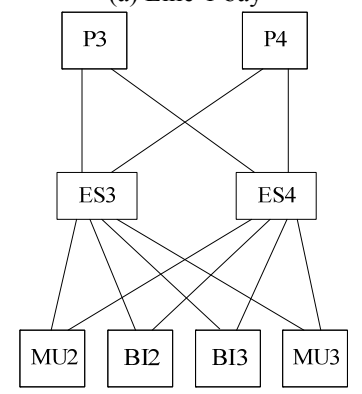

(b) T-1 bay

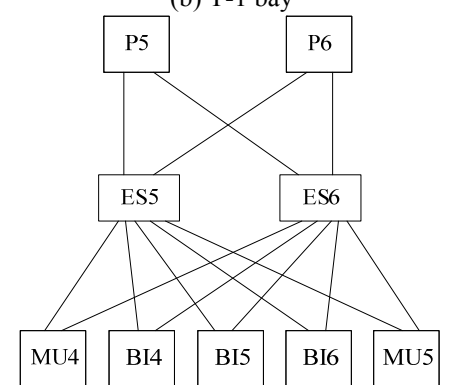

(c) Bus-1 bay

FIGURE III. PROCESS BUS ARCHITECTURES FOR SOLUTION \#1

\section{2) SV and GOOSE LAN Separate (PB solution \#2)}

To guarantee the security and the real-time performance of the protection functions, a widely used process bus solution (PB \#2) is that the GOOLE LAN is separated from the SV LAN. The Protection IEDs are connected to the corresponding circuit breaker IED (or smart terminal) via the GOOSE LAN which achieves the measurement and control of the primary devices such as switches and circuit breakers. Figure 4 shows the process bus architectures for this solution.

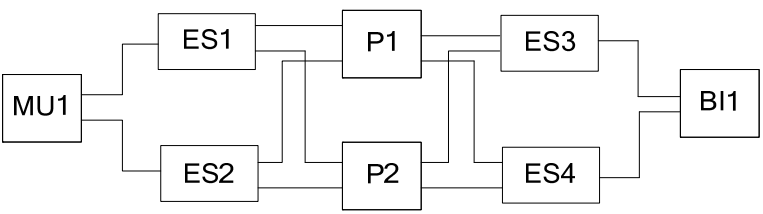

(a) Line-1 bay

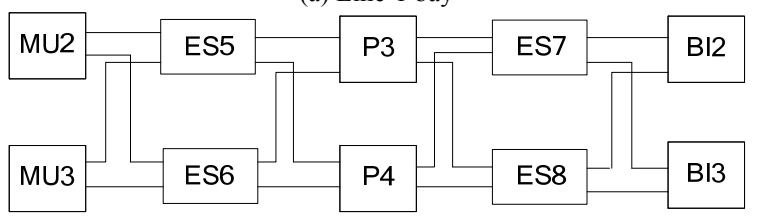

(b) T-1 bay 


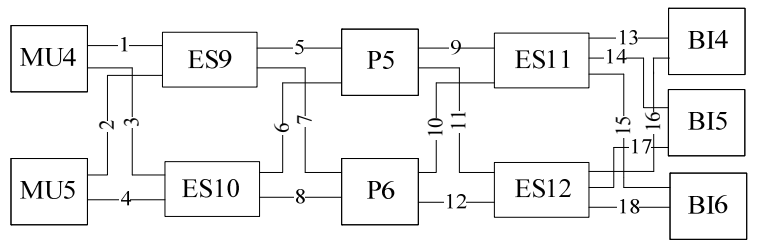

(c) Bus-1 bay

FIGURE IV. PROCESS BUS ARCHITECTURES FOR SOLUTION \#2

\section{RELIABILITY ANALYSIS METHOD}

To evaluate the reliability of process bus related to protection functions, it is assumed that the reliability of the $\mathrm{CT} / \mathrm{PT}$, circuit breakers and the copper wires between process bus devices and primary devices is $100 \%$. This paper only considers the following components,

- Protection IED

- Merging Unit

- Ethernet Switch

- Breaker IED

- Optical Cable

From a reliability point of view, the protection system is considered working when all the MUs have access to their associated protection IEDs as well as the breaker IEDs. For the non-redundant components such as MU and breaker IED, any one of them failure can cause the system failure.

\section{A. Failure Mode of Redundant System}

For redundant system, there are two failure modes to be considered, one for independent and the other for dependent. If the failure modes are independent from each other, the system reliability can be calculated by

$$
R_{S}=\prod_{i=1}^{m}\left[1-\prod_{n \in c_{i}}\left(1-R_{n}\right)\right]=\prod_{i=1}^{m}\left(1-F_{i}\right)
$$

Where, $m$ is the total number of minimum cut sets, $C_{i}$ is the $i^{\text {th }}$ minimum cut set, $R_{n}$ is the reliability for the $n^{\text {th }}$ component, $F_{i}$ is the unreliability for the $i^{\text {th }}$ minimum cut set.

Most of current literatures use the independent failure modes for the reliability analysis of substation communication network. However, the failure modes of redundancy system are not necessarily independent. For example, in Figure 4 (c), the two minimal cut sets, $A=\{O C 1, O C 3\}$ and $B=\{O C 1, O C 2$, ES10\} have common failure events (optical cable 1 failure). If the intersection of A and B is omitted, the calculated reliability will become smaller than the normal because $A \cap B$ has been considered repeatedly. There may be quite a few dependent modes in the redundant system, especially for the parallel redundancy protocol based process bus to achieve the crossbackup functions. Therefore both the dependent and independent modes must be taken into account. With this motivation, the direct disjoint form (DDF) calculation method of network system reliability is considered in this paper.

\section{B. Direct Disjoint Form of Minimal Cut Sets}

For a system with a total of $m$ minimal cut sets, the $i^{\text {th }}$ minimal cut set can be expressed as

$$
C_{i}=\prod_{1 \leq j \leq n} x_{i j} \quad(i=1,2, \cdots, m)
$$

Where, $x i j$ is the basic event, and $n$ is the total number of the basic events in $\mathrm{Ci}$.

The Boolean negation of $\mathrm{Ci}$ is computed as follows

$$
\overline{C_{i}}=\overline{X_{i 1} x_{i 2} \cdots X_{i n}}=\bar{X}_{i 1}+x_{i 1} \bar{X}_{i 2}+\cdots+\left[\prod_{k=1}^{n-1} x_{i k}\right] \bar{X}_{i n}
$$

It is usually expressed using the lower triangular matrix as

$$
\begin{aligned}
& X=\left[\begin{array}{cccc}
\overline{x_{i 1}} & & & \\
x_{i 1} & \overline{x_{i 2}} & & \\
\vdots & \vdots & \ddots & \\
x_{i 1} & x_{i 2} & \cdots & \bar{x}_{i n}
\end{array}\right] \\
& \bar{C}=\left[\sum_{j=1}^{n} \prod_{k=1}^{j-1} x_{j k}\right] x_{j j}
\end{aligned}
$$

The failure mode for a system is the union of all the minimal cut sets

$$
T=\bigcup_{i=1}^{m} C_{i}
$$

Rewrite (5) in the direct disjoint form

$$
T=C_{1}+\bar{C}_{1} C_{2}+\cdots+\left[\prod_{i=1}^{m-1} \bar{C}_{i}\right] C_{m}
$$

Define

$$
K_{i}=\left\{\begin{array}{cc}
C_{1} & , i=1 \\
\left.\prod_{j=1}^{i-1} \overline{C_{j}}\right] C_{i} & , i>1
\end{array}\right.
$$

The system unreliability is the probability of the top event

$$
F_{S}=P(T)=\sum_{i=1}^{m} P\left(K_{i}\right)
$$

It is observed that equation (8) has a total of $m$ items and each item requires the Boolean operation for one or more minimal cut sets, which may lead to complicated calculation for complex system. 


\section{Approximate Algorithm for Reliability Calculation}

Obviously, if the failure probabilities of components tend to be small, the intersection probability of multi minimal cut sets will become very small and can be negligible. Whereas, if the failure probabilities of components are comparatively high (more than 0.5), for (7),

$$
C_{i}=K_{i} / \prod_{j=1}^{i-1} \overline{C_{j}} \Rightarrow P\left(C_{i}\right)>P\left(K_{i}\right)
$$

The above equation is valid if $i$ is large enough, and hence it is enough to consider the crucial minimal cut sets for approximation calculation when the system has a large number of minimal cut sets. For complex systems, the crucial minimal cut sets can be obtained by the following process

1) Arrange the basic events from the smallest to the largest MTTF. If the MTTF of two basic events is equal, the one with comparatively less availability is preferable considered.

2) The minimal cut set is made of basic event. According the order of basic events, consider the combination of basic events from less to more. It is required that the obtained minimal cut sets include all the primary events, and should be independent from each other as many as possible.

3) Assume that the total number of the obtained minimal cut sets and the communication link of process bus system are $m$ and $n$ respectively. Under second condition, if $m<n$, continue to find the remaining minimal cut sets until $m=n$ ' or all the minimal cut set is determined; If $m \geq n$, the crucial minimal cut sets have been obtained.

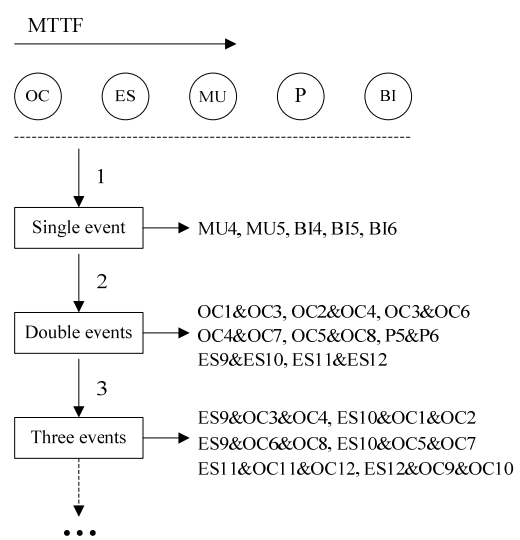

FIGURE V. ENUMERATION PROCESS FOR THE CRUCIAL MINIMAL CUT SETS

For the small transmission substation, the crucial minimal cut sets can be obtained by using the enumeration method. Take the bus protection in Figure 4 (c) for example. It is composed of 29 components that lead to 229 (or 536870912) different combinations to evaluate. However, it is no need considering all the minimal cut sets for approximation calculation. The crucial minimal cut sets can be enumerated by using the presented method as shown in Figure 5.

It is observed that the failure model of the bus protection system contains 19 crucial minimal cut sets, and most of them are not independent. The DDF calculation method should be considered for this type redundant architecture, otherwise the calculation error of reliability will become very large.

It is very important to note that the disjoint process of failure (or success) modes is an essential part for the reliability calculation, i.e. current reliability calculation methods such as RBD, Markov, and Fault Tree have the same complexity with the consideration of dependent modes.

\section{Implementation}

For simplicity, the DDF-based reliability algorithm is developed by using the MATLAB tool. Figure 6 shows the program flow chart of reliability calculation. The fault tree is constructed from a failure point of view, ant the minimal cut sets (MCS) are calculated using the forward "bottom-up" method [16] in combination of the approximation algorithm.

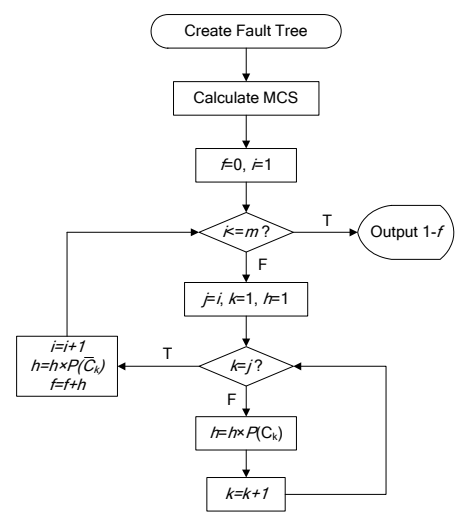

FIGURE VI. IMPLEMENTATION OF THE DDF-BASED RELIABILITY ALGORITHM

\section{ANALYSIS}

\section{A. Reliability Index}

For qualitative and quantitative analyses, the basic assumption is that all the electronic components of the same type have the same failure rate $(\lambda)$ and the repair rate $(\mu)$, and the failures of the basic events obey one-parameter exponential distribution. The reliability function for single component is calculated as follows,

$$
R(t)=e^{-\lambda t}=1-F(t)
$$

The probability of the ith minimal cut set is given by the probability of the intersection of the $n$ failure events

$$
P\left(C_{i}\right)=\prod_{j=1}^{n} F_{i j}(t)
$$

Mean time to failure (MTTF) and mean time to repair (MTTR) are calculated as follows 


$$
M T T F=\frac{1}{\lambda}, \quad M T T R=\frac{1}{\mu}
$$

Availability for a component is given by

$$
A=\frac{\mu}{\mu+\lambda}
$$

MTTF of overall system is calculated as follows

$$
M T T F=\int_{0}^{+\infty}\left[1-F_{S}(t)\right] d t=\int_{0}^{+\infty} R_{S}(t) d t
$$

Similarly, the system availability can be computed by equation (8), if the component reliability is replaced by the component availability.

Table II summarizes the MTTF and MTTR values of each type of element for reliability calculations.

TABLE II. MTTF, MTTR AND AVAILABILITY OF COMPONENTS

\begin{tabular}{|c|c|c|c|c|}
\hline Component & Abbreviation & $\begin{array}{c}\text { MTTF } \\
\text { (years) }\end{array}$ & $\begin{array}{c}\text { MTTR } \\
\text { (hours) }\end{array}$ & $\begin{array}{c}\text { Availability } \\
(\%)\end{array}$ \\
\hline Prot. IED & PI & 150 & 24 & 99.9982 \\
\hline $\begin{array}{c}\text { Merging } \\
\text { Unit }\end{array}$ & MU & 150 & 24 & 99.9982 \\
\hline $\begin{array}{c}\text { Ethernet } \\
\text { Switch }\end{array}$ & ES & 50 & 24 & 99.9945 \\
\hline $\begin{array}{c}\text { Optical } \\
\text { Cable }\end{array}$ & OC & 50 & 24 & 99.9945 \\
\hline $\begin{array}{c}\text { Breaker } \\
\text { IED }\end{array}$ & BI & 150 & 24 & 99.9986 \\
\hline
\end{tabular}

\section{B. Verification}

To validate the presented reliability evaluation method, the comparison of MTTF and availability between the exact and approximate values for 3 bays using PB solution \#2 is conducted (as shown in Table III). It is observed that the availability errors are very small and nearly equal to zero (below 10-6). Since the MTTF is calculated through an integration of reliability from 0 to infinity, it has a larger error as compared to the availability. The number of components determines the complicity of protection systems. The most complex architecture is the bus bay and the simplest is the line bay. It is observed that the MTTF error increases with the complicity of architecture. However, the largest MTTF error introduced by bus bay is $|12.43-12.92| \div 12.43 \approx 3.94 \%<5 \%$, which can be acceptable for the engineering systems evaluation as well as availability.
TABLE III. COMPARISON OF CALCULATION RESULTS BETWEEN EXACT AND APPROXIMATE VALUES

\begin{tabular}{|c|c|c|c|c|c|}
\hline \multirow{2}{*}{ Bay } & \multirow{2}{*}{$\begin{array}{c}\text { Total No. } \\
\text { of } \\
\text { component }\end{array}$} & \multicolumn{2}{|c|}{ MTTF (years) } & \multicolumn{2}{c|}{ Availability (\%) } \\
\cline { 3 - 6 } & Exact & Approx. & Exact & Approx. \\
\hline Line-1 & 20 & 18.12 & 18.20 & 99.9964 & 99.9964 \\
\hline T-1 & 26 & 14.19 & 14.55 & 99.9928 & 99.9928 \\
\hline Bus-1 & 29 & 12.43 & 12.92 & 99.9909 & 99.9909 \\
\hline
\end{tabular}

\section{Discussion}

Table IV shows the comparison of the MTTF and availability between the two PB solutions. It is observed that the PB solution \#1 provides comparatively higher MTTF as compared to $\mathrm{PB}$ solution \#2. The average increase rate of MTTF is calculated as

$$
\Delta=\frac{22.72 \times 3+16.05+13.98-(19.67 \times 3+14.55+12.92)}{19.67 \times 3+14.55+12.92}=13.54 \%
$$

Therefore, the PB solution \#1 can improve the MTTF of each type protection system by an average of about $13.54 \%$.

\begin{tabular}{|c|c|c|c|c|}
\hline \multirow[b]{2}{*}{$\begin{array}{c}\text { Bay } \\
\text { Name }\end{array}$} & \multicolumn{2}{|c|}{ PB solution \#1 } & \multicolumn{2}{|c|}{ PB solution \#2 } \\
\hline & $\begin{array}{l}\text { MTTF } \\
\text { (year) }\end{array}$ & $\begin{array}{c}\text { Availabilit } \\
\text { y (\%) }\end{array}$ & $\begin{array}{c}\text { MTT } \\
\text { F } \\
\text { (year) }\end{array}$ & $\begin{array}{c}\text { Availabilit } \\
\text { y (\%) }\end{array}$ \\
\hline Line-1 & 22.72 & 99.9964 & 19.67 & 99.9964 \\
\hline $\mathrm{T}-1$ & 16.05 & 99.9928 & 14.55 & 99.9928 \\
\hline Bus-1 & 13.98 & 99.9909 & 12.92 & 99.9909 \\
\hline Line-2 & 22.73 & 99.9964 & 19.67 & 99.9964 \\
\hline Line-3 & 22.73 & 99.9964 & 19.67 & 99.9964 \\
\hline
\end{tabular}
However, the two process bus solutions have the same availability for each bay.

TABLE IV. MTTF AND AVAILABILITY OF PROCESS BUS ARCHITECTURES

Table 5 shows the evaluation results for the entire protection system of the small transmission substation. It is observed that the two PB solutions still have the same availability (99.9729). Whereas, the improvement of MTTF by using PB solution \#1 has been reduced to

$$
\Delta=\frac{5.91-5.52}{5.52}=7.05 \%
$$

On the other hand, the system unreliability at 1000 hours of the two process bus solutions are approximately equal, and there is no remarkable difference in reliability and availability between the two solutions except that $\mathrm{PB}$ solution $\# 2$ requires 30 additional components (10 Ethernet switches and 20 fiber cables) for the T1-1 small transmission substation. 
TABLE V. EVALUATION RESULTS FOR THE OVERALL PROTECTION SYSTEMS

\begin{tabular}{|c|c|c|c|c|}
\hline $\begin{array}{c}\text { PB } \\
\text { solution }\end{array}$ & $\begin{array}{c}\text { Total No. of } \\
\text { Components }\end{array}$ & $\begin{array}{c}F_{S}(t) \\
(t=1000 \mathrm{~h})\end{array}$ & $\begin{array}{c}\text { MTTF } \\
\text { (year) }\end{array}$ & $\begin{array}{c}\text { Availability } \\
(\%)\end{array}$ \\
\hline$\# 1$ & 85 & $11.46 \% 0$ & 5.91 & 99.97299 \\
\hline$\# 2$ & 115 & $11.48 \% 0$ & 5.52 & 99.97299 \\
\hline
\end{tabular}

The above results indicate that $\mathrm{PB}$ solution \#1 which combines SV and GOOSE LAN provides comparatively higher MTTF, but has no obvious effect on increasing the availability and the reliability of overall protection systems. PB solution \#2 requires more Ethernet switches and fiber cables to achieve the process bus communication. However, since the SV LAN is separated from GOOSE LAN, the large SV traffic cannot affect the transmission latency of GOOSE messages, which ensures the high-speed and secure transmission of the critical information such as trip signals and the control commends.

Considering the different advantages of the two PB solutions, some suggestions for process bus deployment are presented as follows.

1) $\mathrm{PB}$ solution $\# 1$ is more suitable for line protection as it only requires the voltage and current of a feeder. The MU and breaker IEDs can be doubled to improve the reliability of protection system.

2) In the small substation, PB solution $\# 1$ is preferably considered for the transformer and bus protection. PB solution \#2 should be considered for 3 winding transformer in the substation with $3 / 2$ connection mode and the bus connected to more than 4 feeders.

In the large substation, $\mathrm{PB}$ solution \#2 is more suitable for transformer and bus protection. The MU and breaker IEDs should be doubled to enhance the reliability of protection system.

\section{CONCLUSION}

Based on PRP principle, two typical process solutions for protection system are discussed for a typical T1-1 small transmission substation. Considering the influence of common failure events on the calculation precision of reliability, the direct disjoint form calculation method of network system reliability is presented for the transmission substation. To reduce the calculation complicity, an approximation algorithm is presented and demonstrated for the bus protection system. The comparison results shows that the presented calculation method is simple and effective for reliability evaluation of process bus. From a reliability point of view, the PB solution \#2, which separates the SV LAN from GOOSE LAN, provides smaller MTTF, because the additional component requirements decrease the reliability of protection system. Whereas, PB solution \#1, which combines the SV and GOOSE LAN, makes a small improvement in MTTF and saves half of the cost of Ethernet switches. However, PB solution \#2 can provide the secure and high-speed network environment for GOOSE transmission. It is recommended to consider the network performance for PB solutions \#1, especially, evaluate the impact of SV traffic on the transmission of GOOSE messages. Finally, a global evaluation of process bus [7] needs to be conducted to produce a more accurate result for the practical requirement of substation.

\section{REFERENCES}

[1] IEC Standard for Communication network and systems in substations, IEC 61850, 2003.

[2] J. C. Tan, W. P. Luan, "IEC 61850 based substation automation system architecture design," in IEEE International Conference on Power and Energy Society General Meeting, 2011.

[3] IEC Standard for Highly available automation networks, IEC 62439, 2007.

[4] L.Andersson, K.P. Brand, C. Brunner, and W. Wimmer, "Reliability investigations for SA communication architectures based on IEC 61850," in Proc. IEEE Power Tech., Aug. 2005.

[5] M. Kanabar and T. Sidhu, "Reliability and Availability Analysis of IEC 61850 Based Substation Communication Architectures," in The 2009 IEEE PES General Meeting, Calgary, Canada, July 2009.

[6] K. Jiang, C. Singh, "Reliability modeling of ALL-Digital Protection System Including Impact of Repair," IEEE Trans. Power Del., vol.25, no. 2, pp. 579-586, Apr. 2010

[7] J. C. Tournier, T. Werner, "A Quantitative Evaluation of IEC61850 Process Bus Architectures," in IEEE International Conference on Power and Energy Society General Meeting, 2010

[8] J. Mo, J. C. Tan, P. A. Crossley, Z. Q. Bo, A. Klimek, "Evaluation of process bus reliability," in IET International Conference on Developments in Power System Protection, 2010.

[9] P. Zhang, L. Portillo, and M. Kezunovic, " Reliability and Component Importance Analysis of All-Digital Protection Systems," in Proc. IEEE Power System Conf. and Expo., pp. 1380-1387, 2006.

[10] M. Khademi, "Reliability of telecommunications for bulk power system teleprotection," IEEE Trans. Power Del., vol. 12, no. 2, pp. 601-606, Apr. 1997.

[11] B. L. Deusrmeyer, "A new approach for network reliability analysis," IEEE Trans. Reliab., vol. R-31, no. 4, pp. 350-354, Oct. 1982.

[12] R. Suresh, "A cutset approach to reliability evaluation in communication networks," IEEE Trans. Reliab., vol. R-31, no. 5, pp. 428-431, Dec. 1982.

[13] J. Walrand and P. Varaiya, High-Performance Communication Networks. San Francisco, CA: Morgan Kaufmann, 2002.

[14] Z. H. Dai, Z. P. Wang, and Y. J. Jiao, "Reliability Evaluation of the Communication Network in Wide-Area Protection," IEEE Trans. Power Del., vol.26, no. 4, pp. 579-586, Oct. 2011.

[15] T. D. Jin, Complex System Reliability Analysis and Optimization Considering Component Reliability Estimation Uncertainty, New Jersey: The State University of New Jersey, 2001.

[16] J. B. Fussell, "How to hand-calculate system safety and reliability characteristics," IEEE Trans. Rel., vol.24, no. 3, pp. 169-174, Apr. 1975. 